\title{
Impacto da deficiência de vitamina D na prevalência de cárie precoce na infância: um estudo de revisão
}

\author{
Impact of vitamin D deficiency on the prevalence of early childhood \\ caries: a review study
}

Valdeci Elias dos Santos Junior" Aronita Rosenblatt*

\section{Resumo \\ Objetivo: o objetivo deste trabalho é realizar um levan- tamento dos estudos publicados de 2004 a 2014 sobre o impacto da deficiência de vitamina $D$ na prevalência de cárie precoce na infância. Método: a estratégia de busca das publicações incluiu as bases de dados Medli- ne, Lilacs e Pubmed. A seleção dos artigos foi feita com base nos seguintes critérios: ter sido escrito em inglês, espanhol ou português; apresentar resumo estruturado e abordar aspectos que relacionem a deficiência de vi- tamina D com cárie dentária em crianças. Após a análi- se crítica dos resumos, sete estudos foram selecionados. Considerações finais: há clara associação entre defici- ência de vitamina $D$ e cárie precoce na infância. Além disso, a literatura atual sugere que a suplementação de vitamina $D$ pode reduzir o risco do desenvolvimento de lesões cariosas.}

Palavras-chave: Cárie dentária. Criança. Vitamina D. Deficiência nutricional.

\section{Introdução}

Cárie precoce na infância é um problema de saúde pública comum em países em desenvolvimento, nos quais a desnutrição ainda é um desafio ${ }^{1}$. Esse padrão de cárie desenvolve-se muito rapidamente em pré-escolares, afetando as superfícies dos dentes que são naturalmente livres de cárie ${ }^{2,3}$. Considerando que a doença é influenciada pelo contexto social, essa discussão vai além dos limites das questões de saúde bucal e adentra os limites da equidade social ${ }^{4}$.

Fatores de risco perinatais podem resultar em condições de saúde bucal desfavoráveis, considerando que crianças com baixo peso ao nascer tendem a desenvolver defeitos de esmalte, o que facilita a adesão e colonização de bactérias cariogênicas. A literatura atual ${ }^{5,6}$ indica que existe uma forte associação entre defeitos do esmalte, prematuridade e baixo peso ao nascer. 
A vitamina $\mathrm{D}$ regula os níveis de cálcio e desempenha papel fundamental no desenvolvimento craniofacial e na manutenção de uma boa saúde oral ${ }^{5}$. A deficiência de vitamina $\mathrm{D}$ durante os períodos de desenvolvimento dos dentes pode resultar em defeitos de desenvolvimento ${ }^{6}$, incluindo hipoplasia do esmalte, um fator de risco para o desenvolvimento de cárie precoce na infância. A vitamina $\mathrm{D}$ está associada às duas principais doenças bucais, cárie $\mathrm{e}$ doença periodontal ${ }^{7-9}$. De modo geral, maiores níveis sorológicos dessa substância acarretam em melhores condições de saúde bucal ${ }^{7,10,11}$. A vitamina D também tem importante papel imunológico por induzir a produção de peptídeos antimicrobianos contra patógenos orais ${ }^{11,12}$.

Assim, o objetivo deste estudo é analisar a associação entre a deficiência de vitamina $\mathrm{D}$ e a cárie precoce na infância por meio de um estudo exploratório de revisão sistematizada da literatura.

\section{Materiais e método}

A investigação dos artigos incluídos neste estudo foi realizada nas bases de dados Medline, Lilacs e Pubmed, com a utilização dos seguintes descritores de saúde em inglês: Early childhood caries e vitamin $D$.

Os critérios de inclusão dos artigos foram: ter sido escrito em inglês, espanhol ou português, apresentar resumo estruturado e resultados que abordassem o impacto da deficiência de vitamina $\mathrm{D}$ na prevalência de cárie precoce na infância e ter sido publicado no período entre 2004 e 2014.

\section{Resultados}

Foram encontrados nove estudos, dos quais sete preencheram todos os critérios de inclusão. No entanto, desses sete artigos, um estudo piloto, por ser contemplado em um estudo de maior duração, foi excluído da presente revisão de literatura.

A Tabela 1 apresenta a relação dos estudos incluídos nesta revisão, bem como seu ano de realização, tipo de estudo e conclusão.

Tabela 1 - Estudos inseridos conforme ano, tipo de estudo e conclusão

\begin{tabular}{|c|c|c|c|}
\hline Autores & Ano & Tipo de estudo & Conclusão \\
\hline Schroth RJ et al. ${ }^{13}$ (2014) & 2014 & Coorte prospectivo & $\begin{array}{l}\text { Os níveis de vitamina D maternal têm } \\
\text { influência sobre o desenvolvimento de } \\
\text { cárie precoce na infância }\end{array}$ \\
\hline Davit-Béal T et al. ${ }^{14}$ (2014) & 2014 & Relato de caso & $\begin{array}{l}\text { Os casos apresentavam cárie e } \\
\text { raquitismo por deficiência de vitamina D }\end{array}$ \\
\hline Schroth RJ et al. ${ }^{15}$ (2013) & 2013 & Caso controle & $\begin{array}{l}\text { Crianças com cárie precoce na infância } \\
\text { tiveram índices mais baixos de vitamina D }\end{array}$ \\
\hline Hujoel PP16 (2013) & 2013 & Revisão sistemática e meta-análise & $\begin{array}{l}\text { A vitamina D pode prevenir cárie } \\
\text { precoce na infância. }\end{array}$ \\
\hline Turnbull B et al. ${ }^{17}$ (2007) & 2007 & Artigo de revisão & $\begin{array}{l}\text { Expressa o impacto negativo da condição } \\
\text { nutricional desfavorável. }\end{array}$ \\
\hline Schroth RJ et al. ${ }^{18}$ (2005) & 2005 & tranversal & $\begin{array}{l}\text { A suplementação de vitamina D não se } \\
\text { mostrou eficaz na redução de defeitos de } \\
\text { esmalte e cárie. }\end{array}$ \\
\hline
\end{tabular}

\section{Discussão}

Sugere-se que os eventos da vida humana podem alterar os mecanismos biológicos estáveis e transformá-los em um legado genético, um processo chamado de incorporação biológica ${ }^{19}$. Sob essa perspectiva, fatores etiológicos primários para cárie, como a associação de alta ingestão de açúcar ${ }^{20,21}$, a má higiene oral ${ }^{22-24}$, a falta de exposição ao flúor e os distúrbios perinatais relacionados com defeitos de esmalte ${ }^{21,24}$ poderiam induzir tal legado.

Atualmente, estudos ${ }^{13-18}$ mostram que indivíduos submetidos a níveis baixos de vitamina $\mathrm{D}$ no período pré-natal podem desenvolver defeitos de esmalte, que é um fator de risco para o desenvolvimento de cárie precoce na infância. Além disso, outros fatores perinatais, como baixo peso e prematuridade gestacional, mostraram-se fatores de risco para o desenvolvimento desse agravo na infân$\operatorname{cia}^{24,25}$.

Portanto, os profissionais de saúde devem estar cientes das potenciais deficiências nutricionais que submetem as crianças a riscos de desenvolver cáries severas. É importante considerar que a cárie precoce na infância e o estado de má nutrição estão intimamente associados ${ }^{11,26}$. 
A expressão vitamina D é uma denominação empregada para designar os diversos compostos que possuem a propriedade de regulação de níveis de cálcio e fósforo. Os mais importantes são o ergocalciferol e o colecalciferol, embora cerca de onze compostos expressem atividade de vitamina $\mathrm{D}$. As vitaminas $\mathrm{D}$ são formadas a partir de suas pró-vitaminas no organismo, ativadas pela ação da radiação ultravioleta da luz solar. Importantes atividades da vitamina D, particularmente em intestino, rins e ossos, colocam-na na condição de um hormônio para a manutenção da homeostase do cálcio e do fosfato. Alterações ou deficiências no seu mecanismo de ativação e de controle da absorção resultam em distúrbios orgânicos, podendo evoluir para importantes patologias como o raquitismo, osteomalácia e cárie precoce na infância ${ }^{27}$.

$\mathrm{Na}$ ausência da vitamina $\mathrm{D}$, a absorção do cálcio é drasticamente reduzida. Com a inadequada absorção do cálcio, há sua mobilização de depósitos ósseos que pode conduzir, especialmente em crianças, a sérias doenças ósseas metabólicas, como o raquitis$\mathrm{mo}^{28}$. Além disso, a fase de calcificação da odontogênese também pode ser seriamente afetada com essa deficiência de vitamina $\mathrm{D}$, gerando dentes hipomineralizados ${ }^{29}$, mais susceptíveis a doença cárie.

Embora evidências científicas sugiram que suplementação de vitamina $\mathrm{D}$ pode prevenir o surgimento de lesões de cáries, grande parte dessas pesquisas tem sido negligenciada ${ }^{8,16}$. Recentemente, uma meta-análise relatou que a suplementação de vitamina $\mathrm{D}$, em concentrações entre $75-100 \mathrm{nmol} / \mathrm{L}$, pode reduzir o risco do desenvolvimento de lesões de cárie ${ }^{11,16}$.

Logo, com o avanço da ciência e a rapidez da divulgação dos achados mais recentes, não seria adequado continuar a referir-se à etiologia da cárie dentária exclusivamente pela intersecção dos seus fatores primários, tais como microbiota, hospedeiro e substrato ${ }^{30}$.

Analisando o processo de transição epidemiológica da cárie dentária na população infantil de países mais desenvolvidos, observa-se um declínio acompanhado de um fenômeno conhecido como polarização, caracterizado por vulnerabilidade social para o desenvolvimento da cárie dentária. Corroborando com isso, verifica-se que uma maior carga da doença acomete populações economicamente menos favorecidas ${ }^{30}$. Portanto, é essencial monitorar a prevalência e a severidade da doença e avaliar os fatores risco, em especial em países em que a desnutrição ainda é presente, como é o caso do Brasil.

\section{Considerações finais}

Com base na pesquisa realizada, é possível afirmar que há uma evidente associação entre deficiência de vitamina $\mathrm{D}$ e cárie precoce na infância, uma vez que a literatura atual sugere que a suplementação de vitamina $\mathrm{D}$ pode reduzir o risco do desenvolvimento de lesões cariosas.

\section{Abstract}

Objective: This study aimed to survey articles, published from 2004 to 2014, about the impact of vitamin $D$ deficiency on the prevalence of early childhood caries. Materials and method: The search strategy included Medline, Lilacs, and Pubmed databases. Articles selection was based on the following criteria: to have been written in English, Spanish or Portuguese; to present structured abstract; and to address issues that relate vitamin $D$ deficiency to dental caries in children. After a critical review of abstracts, seven studies were selected. Final considerations: There is a clear association between vitamin $D$ deficiency and early childhood caries. In addition, current literature suggests that vitamin $D$ supplementation may reduce the risk of developing caries lesions.

Keywords: Dental Caries. Child. Vitamin D. Nutritional Deficiency.

\section{Referências}

1. Petersen PE. The World Oral Health Report 2003: continuous improvement of oral health in the 21st century--the approach of the WHO Global Oral Health Programme. Community Dent Oral Epidemiol 2003; 31suppl 1:3-23.

2. Suckling GW. Development defects of enamel - historical and present day perspectives of their pathogenesis. Adv Dent Res 1989; 3:87-94.

3. Horowitz HS. Research issues in early childhood caries. Community Dent Oral Epidemiol 1998; Suppl 1:67-81.

4. Fearne JM, Bryan EM, Elliman AM, Brook AH, Wlliams DM. Enamel defects in the primary dentition of children born weighing less than 2000g. Br Dent J 1990; 168:433-7.

5. Goodman AH, Martinez C, Chavez A. Nutritional supplementation and the development of linear enamel hypoplasias in children from Tezonteopan, Mexico. Am J Clin Nutr $1991 ; 53: 773-81$.

6. Berdal A, Bailleul-Forestier I, Davideau J, Lezot F. Dento-alveolar bone complex and vitamin D. In: Feldman D, Pike J, Glorieux F. Vitamin D. Burlington: Elsevier Academic Press; 2005. p. 599-607.

7. Schroth R, Jeal N, Kliewer E, Sellers EA. The relationship between vitamin $\mathrm{D}$ and severe early childhood caries: a pilot study. Int J Vitam Nutr Res 2012; 82:53-62.

8. Mellanby M, Pattison C. The action of vitamin D in preventing the spread and promoting the arrest of caries in children. Br Med J 1928; 2:1079-82.

9. Hildebolt CF. Effect of vitamin D and calcium on periodontitis. J Periodontol 2005; 76:1576-87.

10. Dietrich T, Joshipura KJ, Dawson-Hughes B, Bischoff-Ferrari HA. Association between serum concentrations of 25-hydroxyvitamin D3 and periodontal disease in the US population. Am J Clin Nutr 2004; 80:108-13.

11. Grant WB. A review of the role of solar ultraviolet-B irradiance and vitamin $\mathrm{D}$ in reducing risk of dental caries. Dermatoendocrinol 2011; 3:193-8.

12. Hewison M. Vitamin D and the immune system: new perspectives on an old theme. Endocrinol Metab Clin North Am 2010; 39:365-79. 
13. Schroth RJ, Lavelle C, Tate R, Bruce S, Billings RJ, Moffatt ME. Prenatal Vitamin D and Dental Caries in Infants. Pediatrics 2014; 33(5):e1277-84

14. Davit-Béal T, Gabay J, Antoniolli P, Masle-Farquhar J, Wolikow M. Dental complications of rickets in early childhood: case report on 2 young girls. Pediatrics 2014; 133(4):e107781.

15. Schroth RJ, Levi JA, Sellers EA, Friel J, Kliewer E, Moffatt ME. Vitamin D status of children with severe early childhood caries: a case-control study. BMC Pediatr 2013; 13:174.

16. Hujoel PP. Vitamin D and dental caries in controlled clinical trials: systematic review and meta-analysis. Nutr Rev 2013; 71:88-97.

17. Turnbull B, Lanigan J, Singhal A. Toddler diets in the U.K.: deficiencies and imbalances. 1. Risk of micronutrient deficiencies. J Fam Health Care 2007; 17(5):167-70.

18. Schroth RJ, Smith PJ, Whalen JC, Lekic C, Moffatt ME. Prevalence of caries among preschool-aged children in a northern Manitoba community. J Can Dent Assoc 2005; 71(1):27.

19. Hertzman C, Boyce T. How experience gets under the skin to create gradients in developmental health. Annu Rev of Public Health 2010; 31:329-47.

20. Rosenblatt A, Zarzar P. The prevalence of early childhood caries in 12- to 36-month-old children in Recife, Brazil. ASDCJ Dent Children 2002; 69:319-24.

21. Milgrom P, Riedy CA, Weinstein P, Tanner ACR, Manibusan L, Bruss J. Dental caries and its relationship to bacterial infection, hipoplasia, diet, and oral hygiene in 6- to 36-monthold children. Com Dent Oral Epidemiol 2000; 28:295-306.

22. Harris R, Nicoll AD, Adair PM, Pine CM. Risk factors for dental caries in young children: a systematic review of the literature. Com Dent Health 2004; 21(1):71-85.

23. Warren JJ, Weber-Gasparoni K, Marshall TA, Drake DR, Dehkordi-Vakil F, Dawson DV, et al. A longitudinal study of dental caries risk among very Young low SES children. Com Dent Oral Epidemiol 2009; 37:116-22.

24. Targino AGR, Rosenblatt A, Oliveira AF, Chaves AMB, Santos VE. The relationship of enamel defects and caries: a cohort study. Oral Diseases 2011; 17:420-6.

25. Santos Junior VE, Sousa RM, Oliveira MC, Caldas Junior AF, Rosenblatt A. Early childhood caries and its relationship with perinatal, socioeconomic and nutritional risks: a cross-sectional study. BMC Oral Health 2014; 6(14):47.

26. Schroth RJ, Harrison RL, Moffatt ME. Oral health of indigenous children and the influence of early childhood caries on childhood health and well-being. Pediatr Clin North Am 2009; 56:1481-99.

27. Barral D, Barros AC, Araújo RPC. Vitamina D: uma abordagem molecular. Pesq Bras Odontoped Clin Integr 2007; 7(3):309-15.

28. Menoli APV, Fanchin PT, Duarte DA, Ferreira SL, Imparato JCP. Nutrição e desenvolvimento dentário. Publ UEPG Cienc Biol Saude 2003; 9(2):33-40.

29. Guyton AC, Hall JE. Tratado de fisiologia médica. 9. ed. Rio de Janeiro: Guanabara Koogan; 1997. 1014p.

30. Narvai PC, Frazão P, Roncalli AG, Antunes JLF. Cárie dentária no Brasil: declínio, polarização, iniqüidade e exclusão social. Rev Panam Salud Publica/Pan Am J Public Health 2006; 19(6):385-93.

\section{Endereco para correspondência:}

Valdeci Elias dos Santos Junior

Rua São Sebastião 417, 101

54410-500 Jaboatão dos Guararapes-PE, Brasil Telefone: 5581997446105

E-mail: valdeciodonto@gmail.com

Recebido: 16/06/14. Aceito: 26/06/15. 